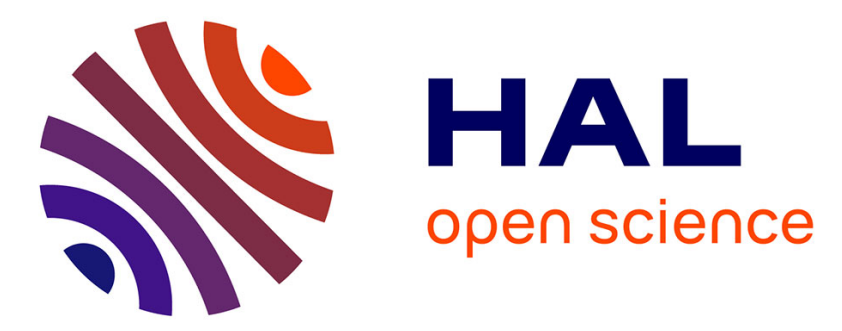

\title{
Les différentes formes de progrès technique et l'évolution économique. Quelques réflexions analytiques à partir de l'étude des industries chimiques \\ Jean-Luc Gaffard
}

\section{- To cite this version:}

Jean-Luc Gaffard. Les différentes formes de progrès technique et l'évolution économique. Quelques réflexions analytiques à partir de l'étude des industries chimiques. Revue d'économie industrielle , 1982, 22 (1), pp.39-46. 10.3406/rei.1982.2070 . hal-01016761

HAL Id: hal-01016761

https://hal-sciencespo.archives-ouvertes.fr/hal-01016761

Submitted on 1 Jul 2014

HAL is a multi-disciplinary open access archive for the deposit and dissemination of scientific research documents, whether they are published or not. The documents may come from teaching and research institutions in France or abroad, or from public or private research centers.
L'archive ouverte pluridisciplinaire HAL, est destinée au dépôt et à la diffusion de documents scientifiques de niveau recherche, publiés ou non, émanant des établissements d'enseignement et de recherche français ou étrangers, des laboratoires publics ou privés.

\section{(ㅇ)(1) $\$$}

Distributed under a Creative Commons Attribution - NonCommercial - NoDerivatives| 4.0 


\title{
Persée
}

http://www.persee.fr

\section{Les différentes formes de progrès technique et l'évolution économique - Quelques réflexions analytiques à partir de l'étude des industries chimiques}

\author{
Jean-Luc Gaffard
}

Revue d'économie industrielle, Année 1982, Volume 22, Numéro 1

p. $39-46$

Voir l'article en ligne

An economic analysis of technical progress should basically aim at the process of change in which the single firm, or the economy as a whole absorb the technical novelty. Furthermore, technical progress may enter the existing productive structure, or on the contrary, chang it significantly. The consequences for the rate of growth, production and unemployment are accordingly very different. This calls in our opinion upon an inductive research needed to assume external consistency. The particular study of the Chemical industry seems with this respect to reveal the ongoing forms of technical progress as well as growth instability to which they are associated. The above research indicate clearly the necessity to define what must be a transition towards new principies of growth that may alter this growth instability. Such a transition needs both time and ressources as much as a selective public policy.

\footnotetext{
Avertissement

L'éditeur du site «PERSEE » - le Ministère de la jeunesse, de l'éducation nationale et de la recherche, Direction de l'enseignement supérieur, Sous-direction des bibliothèques et de la documentation - détient la propriété intellectuelle et les droits d'exploitation. A ce titre il est titulaire des droits d'auteur et du droit sui generis du producteur de bases de données sur ce site conformément à la loi n`98-536 du 1er juillet 1998 relative aux bases de données.
}

Les oeuvres reproduites sur le site «PERSEE » sont protégées par les dispositions générales du Code de la propriété intellectuelle.

Droits et devoirs des utilisateurs

Pour un usage strictement privé, la simple reproduction du contenu de ce site est libre.

Pour un usage scientifique ou pédagogique, à des fins de recherches, d'enseignement ou de communication excluant toute exploitation commerciale, la reproduction et la communication au public du contenu de ce site sont autorisées, sous réserve que celles-ci servent d'illustration, ne soient pas substantielles et ne soient pas expressément limitées (plans ou photographies). La mention Le Ministère de la jeunesse, de l'éducation nationale et de la recherche, Direction de l'enseignement supérieur, Sous-direction des bibliothèques et de la documentation sur chaque reproduction tirée du site est obligatoire ainsi que le nom de la revue et- lorsqu'ils sont indiqués - le nom de l'auteur et la référence du document reproduit.

Toute autre reproduction ou communication au public, intégrale ou substantielle du contenu de ce site, par quelque procédé que ce soit, de l'éditeur original de l'oeuvre, de l'auteur et de ses ayants droit.

La reproduction et l'exploitation des photographies et des plans, y compris à des fins commerciales, doivent être autorisés par l'éditeur du site, Le Ministère de la jeunesse, de l'éducation nationale et de la recherche, Direction de l'enseignement supérieur, Sous-direction des bibliothèques et de la documentation (voir http://www.sup.adc.education.fr/bib/ ). La source et les crédits devront toujours être mentionnés. 


\title{
Les différentes formes de progrès technique et l'évolution économique Quelques réflexions analytiques à partir de l'étude des industries chimiques
}

\author{
Jean Luc GAFFARD \\ Professeur de sciences économiques
}

La montée du chômage dans les économies contemporaines n'a pas été endiguée par l'application des politiques de soutien de la demande globale ; elle a été accentuée quand ont été imposées des politiques de restriction monétaire. C'est que, pour une large partie, ce chômage exprime les difficultés de l'économie à s'adapter aux nouvelles technologies, et pose donc le problème des relations entre l'innovation et l'emploi. L'analyse de ce problème est rendue complexe par le fait qu'elle doit prendre en considération les différentes formes d'interdépendance existant entre les agents économiques et entre les secteurs d'activité dont la connaissance est obtenue grâce à des modèles déductifs véhiculant une image du système économique comme système clos et circulaire, alors même que l'émergence de nouveaux processus de production ou de nouveaux biens et le caractère non déterministe de l'évolution engendrée par des décisions successives des agents économiques appellent une approche séquentielle et une démarche inductive. L'objet de la présente contribution n'est pas de parvenir à élaborer complètement une telle analyse, mais il est de proposer un cadre analytique élémentaire pour interpréter certaines observations empiriques concernant la nature des changements technologiques et les stratégies d'entreprise dans un secteur - les industries chimiques - retenu en raison de sa double caractéristique d'être au cœur du régime de croissance, actuellement perturbé, et d'être directement sinon principalement concerné par les nouvelles technologies, parmi les plus importantes du moment, celles de l'information et celles du vivant.

L'idée qui sera défendue et argumentée est qu'il n'existe pas de relation déterminée entre le progrès technique (ou l'innovation) et l'emploi, et que tout dépend de la façon dont ce progrès technique s'inscrit dans les stratégies d'entreprise. Le progrès technique peut être alternativement créateur et destructeur d'emplois. Actuellement, c'est ce dernier aspect qui prévaut ainsi que le révèle l'étude particulière des industries chimiques ; mais le renversement de tendance reste possible pourvu que les contraintes, qui commandent les stratégies des entreprises, puissent être modifiées sous l'effet de politiques économiques dont l'objectif explicite serait d'assurer la transition vers un nouveau régime de croissance. 


\section{I. - LES DIFFERENTES FORMES DE PROGRÉS TECHNIQUE ET L'EMPLOI}

Le progrès technique peut consister alternativement en des changements que l'on qualifiera de mineurs parce qu'ils n'altèrent pas fondamentalement la structure productive existante et parce que l'économie et les entreprises s'y adaptent complètement presque sans aucun délai, et des changements majeurs pour lesquels l'essentiel réside dans le processus de leur acquisition par l'économie et les entreprises.

Le progrès technique n'altère pas la structure productive existante quand il prend la forme d'une diminution des coefficients de travail (par suite d'une meilleure organisation du travail), d'une diminution des coefficients techniques de production (par le jeu des économies d'échelle) ou de l'introduction d'une machine n'impliquant pas un changement de nature des facteurs ou des produits. Ce type de progrès technique peut avoir des effets positifs ou négatifs sur l'emploi : tout dépend du rapport qui s'établit entre les baisses de coûts unitaires (ou les gains de productivité), qui mesurent ce progrès technique, et l'extension des marchés. S'il existe une forte élasticité des prix par rapport aux coûts et de fortes élasticités directes et croisées des demandes par rapport au prix, alors la diffusion de l'innovation se traduit par une interaction entre un accroissement de l'efficacité de la production, et une extension quantitative et qualitative de la demande, qui est au cœur des mécanismes de croissance de la production et de l'emploi. Dans le cas contraire, ces mécanismes sont rompus. Bien plus le progrès technique est destructeur d'emplois soit immédiatement lorsqu'il aboutit à substituer des machines à des travailleurs pour un volume de production constant, soit à un terme plus ou moins rapproché, à travers les mécanismes de concurrence par l'investissement porteur de ce progrès technique et générateur de surcapacités, donc de licenciements sous la contrainte d'une demande en croissance ralentie. Ainsi dans le cadre d'une certaine structure productive et d'un certain système de préférences le progrès technique apparaît comme l'élément moteur de la croissance de la production et de l'emploi, mais il peut aussi constituer un facteur d'instabilité du régime de croissance dont l'un des aspects est la montée du chômage.

Le progrès technique altère la structure productive quand il implique la construction de nouveaux équipements préalable à la mise en œuvre de nouveaux processus de production qui transforment de nouveaux facteurs en de nouveaux produits. Une transition s'avère alors nécessaire entre l'ancienne structure productive et la nouvelle, transition qui précède le moment où la nouvelle structure productive assurera un régime régulier de croissance de la production et de l'emploi, dans des conditions du même type ou différentes de celles qui ont été décrites plus haut. C'est au niveau de cette période de transition que se pose principalement le problème des relations entre le progrès technique et l'emploi. La rareté des ressources financières disponibles implique, en effet, que la transition n'est possible que si l'on opère un transfert d'une partie de ces ressources des anciens processus de production vers ceux que l'on a décidé d'amorcer et de mettre en œuvre, et cela ne peut pas se faire sans pertes de production et d'emplois, compte tenu du temps réel qui doit s'écouler avant que la nouvelle structure productive ne soit mise en place. 
Quand une politique de régulation macroéconomique ne parvient pas à réduire l'instabilité d'un régime de croissance associé à une structure productive déterminée, l'hypothèse peut être émise que le problème en cause n'est pas un problème de stabilité d'un régime de croissance donné, mais un problème de transition vers un autre régime de croissance. Les mêmes inventions peuvent alors très bien devenir des innovations mineures si elles s'inscrivent dans la structure productive existante à travers certaines stratégies d'entreprises et être un facteur d'instabilité, ou devenir des innovations majeures dès lors qu'elles sont progressivement acquises par ces mêmes entreprises en vue de construire une nouvelle structure productive.

\section{II. - L'INSTABILITÉ DU RÉGIME ACTUEL DE CROISSANCE VUE A TRAVERS LE CAS DES INDUSTRIES CHIMIQUES}

Les industries chimiques ont eu l'un des plus forts taux de croissance de toute l'industrie et ont contribué à entraîner la croissance globale ; actuellement, elles subissent le contrecoup d'augmentations de coûts de production et de ralentissements de la demande, révélant ainsi l'instabilité du régime de croissance. Cette évolution concerne aussi bien la chimie lourde que la production de matières plastiques, la chimie fine ou la chimie des spécialités.

Les processus de production de la chimie lourde (oléfines, aromatiques, amoniac, méthanol) sont des processus continus adaptés aux très grandes quantités, et caractérisés par une forte concentration technique. La forme dominante du progrès technique consiste dans une réduction des investissements et des consommations intermédiaires par tonne produite; elle implique un accroissement de la taille optimale des installations. Cette tendance n'a pas été inversée du fait du changement de la structure des coûts et du ralentissement de la demande ; les entreprises jouent sur cette forme de progrès technique pour tenter d'accroître leur part de marché, mais ce faisant, les capacités de production augmentent dans des proportions que ne justifient pas l'accroissement prévisible de la demande. Le phénomène est particulièrement sensible dans la branche de l'éthylène où l'on prévoit une nouvelle aggravation d'excédents de capacité déjà considérables, ce qui n'a rien d'étonnant quand on sait la taille des nouvelles unités construites (715 000 tonnes par an pour la dernière unité construite par Shell-Chemicals aux Etats-Unis). La recherche d'une plus grande flexibilité des installations visant à les rendre compatibles avec plusieurs sources d'énergie ou de matière ne contredit pas cette conclusion. L'exemple des craquages à la vapeur capables de convertir aussi bien le naphta léger que le gas oil a montré que dans la plupart des cas la recherche de flexibilité signifiait l'addition d'un nouveau foyer et un accroissement de capacité. Ainsi les effets de taille étroitement liés aux effets de l'expérience restent dominants dans l'évolution des industries chimiques et commandent une instabilité révélée par des excédents de capacité. Les effets sur l'emploi ne peuvent être que négatifs. Certes, dans le cas de la chimie lourde le nombre des emplois est indépendant du taux d'utilisation de la capacité en raison du caractère continu des processus techniques. Mais il existe une relation entre l'emploi et la capacité de production de telle sorte que les ajustements de cet emploi, pour être discontinus, peuvent être particulièrement brutaux et massifs. Lorsque, en particulier les excédents de capacité dépassent un certain niveau, l'arrêt et le déclassement de certaines unités de production devient inéluctable, entraînant une forte réduction du nombre des emplois. Une telle perspective est actuellement crédible dans ce domaine des productions chimiques, qui pourraient ainsi 
connaître une évolution comparable à celle qui a été constatée dans les industries sidérurgiques. Cela concerne la chimie lourde mais aussi la production de matières plastiques dès lors que la substitution de ces matières à d'autres matériaux plus traditionnels atteint progressivement une limite qu'atteste le passage d'un taux de croissance annuel moyen de $15 \%$ entre 1955 et 1975 à un taux d'environ $6 \%$ prévu d'ici 1990.

Dans le domaine de la chimie fine et de la chimie des spécialités, les stratégies des entreprises consistent actuellement à conserver, à retrouver ou à constituer un portefeuille de produits permettant une compensation réciproque des fluctuations et donc une certaine stabilité des résultats financiers. Ces stratégies passent par le maintien sur certains marchés et par l'entrée sur de nouveaux marchés ; se situant dans un contexte de croissance ralentie de la demande, elles expriment une exacerbation de la concurrence dont chaque entreprise protagoniste sait qu'elle débouche inévitablement sur des excédents de capacité. Ce risque est d'autant plus grand que les prix pratiqués sont rigides relativement à une demande en croissance lente, d'abord parce que cela bloque toute possibilité d'interaction entre les gains de productivité et l'extension des marchés, ensuite parce que les prix limites autorisent des coûts de production unitaires relativement élevés et donc des barrières à l'entrée relativement peu élevées. Ainsi dans ce domaine de la chimie fine et de la chimie des spécialités le progrès technique s'inscrit dans des stratégies de compétition avec pour simple enjeu l'amélioration de la qualité des produits existants et de la performance des procédés de fabrication. Souvent ces procédés sont discontinus et l'automatisation des enchaînements, qui est l'une des formes concrètes des changements techniques actuels, s'accompagne d'accroissements de capacité de l'ordre de 5 à $15 \%$ et pouvant même, quelquefois, dépasser $30 \%$. A terme, l'excédent prévisible de la production sur la demande ne peut que déclencher des ajustements de production, et de capacités de production (par déclassement des unités) évidemment préjudiciables à l'emploi. Là encore le progrès technique en favorisant la concurrence sur des marchés contraints est destructeur d'emplois. Si l'on considère l'automatisation et l'informatisation, ce ne sont pas des changements techniques qui portent en eux-mêmes la destruction des emplois ; ce sont les conditions dans lesquelles ils sont mis en œuvre qui l'impliquent.

L'alternative aux phénomènes d'instabilité qui viennent d'être observés est l'amorce d'un processus de transition vers une autre structure productive.

\section{III. - LA TRANSITION VERS UNE NOUVELLE STRUCTURE PRODUC- TIVE VUE A TRAVERS DU CAS DES INDUSTRIES CHIMIQUES}

Les nouvelles technologies de l'information et du vivant laissent apparaître la possibilité de construire une nouvelle structure productive susceptible de déterminer un régime de croissance régulière de la production et de l'emploi. Cette nouvelle structure productive aurait cette particularité de rendre compatibles l'accroissement de l'efficacité de la production et celui de la variété des produits ; c'est, du moins ce que révèlent les études de prospective relatives au rôle que peuvent avoir ces technologies dans des processus de production susceptibles d'être mis en œuvre par les entreprises appartenant actuellement au secteur des industries chimiques. 
Le recours à des technologies informatiques doit permettre de concevoir de nouveaux produits. Il en est ainsi de plastiques techniques répondant à des propriétés très spécifiques et dont la fabrication, par intégration des phases de réaction chimique, de formulation (introduction d'additifs à la matière de base) et de transformation par moulage, suppose le recours à la microélectronique et à l'optoélectronique. Il en est ainsi, également, de certains produits pharmaceutiques, étroitement adaptés à leurs objectifs thérapeutiques, qui pourraient être conçus grâce aux procédés de synthèse chimique par ordinateur. Par ailleurs, l'informatisation au niveau de processus discontinus doit pouvoir se concrétiser par la mise au point d'ateliers polyvalents, dont le fonctionnement rendrait techniquement et économiquement efficaces des productions de faible volumes, permettant ainsi de répondre à des demandes très spécifiques et très variées. Ainsi pourraient être réamorcés des mécanismes d'interaction entre baisses de coût et hausses des demandes, favorables à la croissance de la production et de l'emploi. Ce même résultat doit pouvoir être attendu du développement des biotechnologies. En premier lieu celles-ci doivent permettre la création d'une gamme étendue de nouveaux produits dès lors que, virtuellement, toutes les molécules de la chimie organique peuvent être obtenues par des voies biologiques, et qu'il est possible de programmer les microorganismes en fonction des tâches qu'ils doivent accomplir, donc en fonction des propriétés et des caractéristiques requises d'un bien déterminé en vue de son usage final ou intermédiaire. En second lieu, ces mêmes biotechnologies introduisent une flexibilité accrue de l'appareil de production puisqu'il est possible d'utiliser un même ensemble productif pour plusieurs productions distinctes : ainsi un avantage de la synthèse enzymatique est sa souplesse qui fait que par simple changement du substrat on peut modifier le processus et produire alternativement les différents oxydes d'alcènes tels que l'oxyde de propylène qui, par polymérisation, donne le polypropylène, et l'oxyde d'éthylène qui donne le polyéthylène. Là encore la baisse des coûts associée à la flexibilité des équipements peut interagir avec la hausse des demandes y compris celles qui sont limitées en volume, et permettre ainsi la croissance de la production et de l'emploi.

La nouveauté des technologies de l'information et du vivant est qu'elles peuvent engendrer une structure productive où la moindre spécificité des biens d'équipement permettrait de dissocier leur temps d'utilisation de la durée de chaque processus de production élémentaire, de telle sorte qu'il serait possible d'obtenir une grande variété de biens finals ou intermédiaires, en maintenant une utilisation maximale des équipements et en bénéficiant d'économies d'échelle. Cela aurait pour première conséquence un élargissement du champ de l'activité productive analogue à celui qu'a constitué le développement des biens de consommation durables et standardisés ; et c'est cet élargissement qui doit être le facteur déterminant de la création d'emplois.

Cependant, le problème est moins dans les caractéristiques du futur régime de croissance que dans les conditions d'acquisition de changements technologiques d'une aussi grande ampleur. A cet égard il faut souligner que le processus de changement consiste, d'abord, dans une profonde transformation du savoir et du savoir-faire puisqu'en fait de nouvelles disciplines scientifiques et techniques se constituent à la conjonction de l'informatique, de la chimie et de la biologie. De cette transformation il résulte la nécessité d'un redécoupage de l'activité productive et d'une redéfinition de la division du travail à l'intérieur des firmes et entre les firmes. L'exemple des plastiques techniques est significatif de ce point de vue 
puisque leur production suppose que la séparation entre producteurs de matières plastiques et transformateurs de ces mêmes matières n'existe plus, ce qui pose un problème stratégique décisif aux entreprises concernées de l'une et l'autre activité (de l'un et l'autre secteur). Les biotechnologies, d'une manière générale, soulèvent le même type de problèmes. Il existe encore une plus grande incertitude sur les conditions d'acquisition de ce changement technologique par les firmes chimiques pour des raisons qui tiennent à la structure industrielle elle-même, que l'on prenne en considération l'expérience industrielle nécessaire ou la nature des relations interindustrielles. L'hypothèse peut être formulée d'un véritable éclatement de l'actuel secteur des industries chimiques à tel point que les firmes spécialisées dans le génie chimique ou le génie enzymatique fourniraient les consommations intermédiaires de processus biotechnologiques intégrés dans plusieurs autres secteurs industriels. Dès lors, la transition est affaire de temps et de ressources : du temps est nécessaire pour construire les nouveaux processus de production, pour mettre en place les nouvelles structures industrielles, donc avant que la production des nouveaux biens ne devienne effective ; des ressources doivent être dégagées pour financer cette construction et cette mise en place. La rareté de telles ressources en période de récession fait que celles qui seraient affectées à l'acquisition de nouvelles technologies seraient détournées de l'amélioration des processus existants, ce qui ne peut que porter atteinte à leur compétitivité et occasionner une diminution de la production et du nombre des emplois. Aussi pour autant que la transition s'avère nécessaire sinon inéluctable, l'instabilité du régime de croissance antérieur et le temps réel associé à cette transition appellent une intervention appropriée des pouvoirs publics.

\section{IV. - LES OBJECTIFS DE LA TRANSITION ET LES INSTRUMENTS DE LA POLITIQUE INDUSTRIELLE}

Le choix d'aider à la transition vers une nouvelle structure productive implique que la politique industrielle ne se réduise pas à favoriser la compétitivité d'entreprises engagées sur des créneaux ou des filières de production qui correspondent à l'ancienne structure. La politique industrielle doit avoir pour objectif de créer un environnement qui induise les entreprises à mettre en œuvre des stratégies aboutissant à l'acquisition des changements techniques radicaux. Pour autant, elle ne doit pas ignorer certaines exigences afférant à la compétitivité des processus existants, dès lors que l'élimination de ces derniers signifierait une phase de dépression socialement inacceptable même si cette dépression doit déboucher sur la transition recherchée. Le cas des industries chimiques devrait encore nous permettre d'orienter l'analyse.

Dans les industries chimiques, il convient de distinguer la chimie de base, en fait peu susceptible d'être radicalement transformée par les nouvelles technologies, des autres activités chimiques directement intéressées par le processus de changement. Pour les entreprises du secteur, la transition doit alors procéder d'une rationalisation de la chimie de base permettant de dégager des ressources pour une réelle transformation des autres activités.

La chimie de base subit des excédents de capacité qui sont la conséquence des stratégies des firmes exprimant aussi bien certaines caractéristiques techniques (spécificité et grande taille des équipements, prédominance des économies d'échelle) que les contraintes économiques (état des coûts et de la demande) ou institutionnelles (organisation du secteur). La rationalisation de cette activité 
consiste à limiter les excédents de capacité et le gaspillage de ressources, que cela représente, en modifiant ces caractéristiques et ces contraintes. Ainsi,dans le cas d'espèce, la politique industrielle doit elle tendre à assurer pour toutes les entreprises un accès égal aux différentes sources d'énergie et de matière afin précisément d'éviter des stratégies d'accroissement des parts des marchés articulées sur des efforts de spécialisation autour de sources particulières (gaz, pétrole, charbon ou électricité nucléaire), afin, aussi, de rendre ces sources plus complémentaires que substituables. La politique industrielle doit, en outre, comprendre des mesures favorisant des restructurations par transferts d'actifs de façon à modifier les contraintes de coût : c'est dans ce sens qu'il faut comprendre l'acquisition d'actifs pétrochimiques par des firmes pétrolières dont l'objectif général est de dégager la valeur ajoutée non pas au niveau de la vente des produits pétroliers mais plus en aval des processus de production, et ceci afin de déserrer la contrainte de coût de la matière première dans les stratégies industrielles et de favoriser la diffusion des baisses de coût enregistrées à chaque étape des différentes filières. La politique industrielle doit enfin aider à une meilleure adaptabilité de l'appareil de production aux fluctuations quantitatives et qualitatives de la demande. C'est en fonction d'un tel objectif qu'il convient d'examiner la possibilité d'un nouveau développement de la chimie du charbon dans la mesure où celle-ci est très flexible au sens où près des trois-quarts du coût de l'investissement concerne la phase de gazéification au terme de laquelle apparaissent les petites molécules et au-delà de laquelle toutes les possibilités de production de molécules complexes restent ouvertes.

La chimie des matières plastiques, la chimie fine et la chimie des spécialités sont le lieu où peuvent s'opérer des transformations radicales. Ces transformations ne peuvent pas être décidées sans que les ressources correspondantes soient dégagées et sans une réduction des effets d'une incertitude inhérente à une transition dont le point d'arrivée n'est pas connu. L'objectif de la politique industrielle est de faire en sorte que de telles conditions puissent être réunies. De ce point de vue, l'instrument le plus approprié semble être le grand programme public faisant appel, pour la réalisation de produits déterminés de haute technologie, à la production et aux services des entreprises. Celles-ci disposent ainsi de ressources destinées directement au financement de la conception et de la fabrication de nouveaux biens et de nouveaux processus de production ; elles engagent un processus d'acquisition des innovations qui, parvenu à son terme, a toutes chances de dépasser qualitativement et quantitativement les objectifs particuliers du programme qui a initié ce processus. Dans le domaine des biotechnologies le nombre de produits qu'il est possible de fabriquer est très grand et dans les conditions économiques actuelles tout choix est impossible ; l'avantage d'un programme public serait d'imposer un choix initial sur lequel s'articuleraient nécessairement plusieurs séquences de décisions et d'événements sans que l'on puisse déterminer a priori laquelle sera suivie.

Le cas des industries chimiques illustre ce qui pourrait être une politique industrielle axée sur l'objectif de transition. En premier lieu elle doit pouvoir limiter les mécanismes d'instabilité associés à la recherche des économies d'échelle sur des marchés limités, et, de ce point de vue, elle ne peut que s'inscrire dans le cadre d'une concertation européenne sinon internationale visant à l'organisation des marchés et à la rationalisation des productions. Il s'agit aussi bien de bloquer des mécanismes de généralisation de la crise qui ne manqueraient pas de se développer à partir d'une chute brutale et massive des emplois industriels, que d'évi- 
ter un gaspillage de ressources et de les orienter vers la construction de la nouvelle structure productive. En second lieu, cette politique industrielle doit être de type horizontal au sens où elle ne doit pas s'inscrire dans le découpage actuel de la structure productive, de manière à aider à son changement. Il s'agit de déclencher des processus d'acquisition de l'innovation sans pour autant en déterminer tous les résultats ; il s'agit de faire en sorte que le progrès technique s'inscrive " positivement » dans les stratégies d'entreprise. Pour cela il faut que ces entreprises disposent de ressources et les affectent à des programmes de développement des nouvelles technologies.

Dans le domaine de l'évolution, la science économique doit étudier, suivant l'expression de N. Georgescu-Roegen, le processus par lequel sont créés de nouveaux moyens économiques, de nouveaux buts économiques et de nouveaux rapports économiques, simplement parce que l'émergence de la nouveauté est au cœur du processus économique. Elle ne peut donc se satisfaire d'une démarche purement déductive associée à une vision du système économique comme système clos et circulaire ; elle doit procéder d'une démarche partiellement inductive dont on a voulu ici donner quelques éléments en partant d'une expérience concrète particulière, celle des industries chimiques, pour induire des réflexions analytiques sur l'instabilité, la transition et les politiques industrielles. 